\title{
Moments Inequalities for NBRUL Distributions with Hypotheses Testing Applications
}

\author{
M. A. W. Mahmoud \\ Al-Azhar University
}

\author{
R. M. EL-Sagheer \\ Al-Azhar University
}

\author{
W. B. H. Etman \\ Al-Azhar University
}

\begin{abstract}
In this paper, moment inequalities for the new better than renwal used in Laplace transform order (NBRUL) class of ageing distributions are derived. This inequalities demonstrate that if the mean life is finite, then all higher order moments exist. A new test for exponentiality versus NBRUL can be constructed using thes inequalities. Pitman's asymptotic efficiencies and critical values of the proposed test are calculated and tabulated. The powers of this test are estimated for some famously alternatives distributions in reliability such as Linear failure rate, Weibull and gamma distributions. Finally, examples in different areas are used as a practical applications of the proposed test.
\end{abstract}

Keywords: classes of life distributions, NBRUL, moments inequalities, life testing.

\section{Introduction}

Classes of life distributions are defined to classify the life distributions according to their aging properties. The definitions of these classes helped statisticians to define the test statistics. The test statistics are defined based on definition of the classes. The main aim of constructing new tests is to gain higher efficiencies. Many authors proposed tests for exponentiality versus some classes of life distributions based on the moment inequalities. Testing exponentiality against IFR, NBU and NBUE based on moment inequalities have been studied by (Ahmad 2001); (Ahmad and Mugdadi 2004) constructed the tests of NBUC, IFRA and DMRL depends on the moment inequalities, while testing NRBU and RNBU based on moment inequalities have been studied by (Mahmoud, EL-arishy, and Diab 2003). Using the moment inequalities of the class NBUL, (Mahmoud, Diab, and Kayid 2009) constructed a test statistic for testing exponentiality versus this class.

In this paper our theme formulates a new test statistic for testing exponentiality against NBRUL class based on the moment inequalities and discuss this test. The main classes of life distributions which have been introduced in the literature are based on new better than used (NBU), new better than used failure rate (NBUFR), new better than average failure rate (NBAFR), new better than used renewal failure rate (NBURFR), new better than used average renewal failure rate (NBARFR), new better than renewal used (NBRU) and exponential better than used in Laplace transform order (EBUL). Testing exponentiality against some classes of life distributions has been introduced by many researchers from many points of views. For more details one can refer to (Bryson and Siddiqui 1969), (Deshpande, Kochar, and Singh 1986), (Abouammoh and Ahmed 1988, 1992), (Abouammoh, Abdulghani, and 
Qamber 1994), (Mahmoud, Moshref, and Mansour 2015), (Kumazawa 1983), (Ahmad 1994, 2001). (Abouammoh and Newby 1989), (Mahmoud and Abdul Alim 2002, 2003, 2008), (Ahmad, Alwasel, and Mugdadi 2001), (Abu-Youssef 2009), (Ismail and Abu-Youssef 2012), (Mahmoud and Rady 2013). Recently (Atallah, Mahmoud, and Al-Zahrani 2014) developed a new method for testing exponentiality which is more general and flexible than goodness approach.

The rest of this paper can be organized as follows, Section 2 gives a brief knowledge about renewal classes. In Section 3 moment inequalities for the NBRUL class are developed. In Section 4, Testing exponentiality against NBRUL is proposed based on moment inequalities. In Section 5, Pitman's asymptotic efficiency (PAE) of the test for several common alternatives will be considered. In Section 6, Monte Carlo null distribution critical points from the null distribution for sample size $n=5(5) 35 ; 39 ; 40(5) 50$. In section 7, The power estimate for the test are calculated. Finally, the application of the proposed test for real data sets are discussed in Section 8.

\section{Renewal classes}

Let $T$ be a random variable represents life time of a device (system or component) with a continuous life distribution $F(t)$. Upon arising the failure of the device, it can be substituted by a sequence of mutually independent devices which are identically distributed with the same life distribution $F(t)$. The following stationary renewal distribution constitutes the remaining life distribution of the device under operation at time $t$.

$$
W_{F}(t)=\mu_{F}^{-1} \int_{0}^{t} \bar{F}(u) d u, \quad t \geq 0,
$$

where $\mu_{F}=\mu=\int_{0}^{\infty} \bar{F}(u) d u$.

It is easy to show that

$$
\overline{W_{F}}(t)=\mu_{F}^{-1} \int_{t}^{\infty} \bar{F}(u) d u, \quad t \geq 0 .
$$

For extra details, see (Barlow and Proschan 1981), (Abouammoh and Ahmed 1988, 1992). Now we need to present the definitions of the NBRU (NWRU) and NBRUL (NWRUL) classes of life distributions.

Definition 2.1. (Abouammoh et al. 1994) If $X$ is a random variable with survival function $\bar{F}(x)$, then $X$ is said to have new better (worse) than renewal used property, denoted by NBRU (NWRU), if

$$
\overline{W_{F}}(x \mid t) \leq(\geq) \bar{F}(x \mid 0), \quad x \geq 0, t \geq 0,
$$

or

$$
\overline{W_{F}}(x+t) \leq(\geq) \overline{W_{F}}(t) \bar{F}(x), \quad x \geq 0, t \geq 0 .
$$

Depending on the definition (2.1), (Mahmoud, EL-Sagheer, and Etman 2016) defined a new class which is called new better (worse) than renewal used in Laplace transform order NBRUL (NWRUL) as follows

Definition 2.2. $X$ is said to be NBRUL (NWRUL) if

$$
\int_{0}^{\infty} e^{-s x} \overline{W_{F}}(x+t) d x \leq(\geq) \overline{W_{F}}(t) \int_{0}^{\infty} e^{-s x} \bar{F}(x) d x, \quad x, t, s \geq 0 .
$$

It is obvious that NBRU $\Rightarrow$ NBRUL $\Rightarrow$ NBRUE.

\section{Moments inequalities}

In this section, the moment inequalities for NBRUL class are established. 
Theorem 3.1. Let $F$ be NBRUL life distribution such that all moments exist and finite then for integers $r \geq 0$ and $s \geq 0$. Then

$$
\begin{aligned}
\frac{\mu_{(r+2)}}{s(r+1)(r+2)}[1-\zeta(s)] \geq & \frac{-(-1)^{r} r !}{s^{r+2}}\left[\mu_{F}-\frac{1}{s}(1-\zeta(s))\right] \\
& +\frac{r !}{s^{r+1}} \sum_{i=0}^{r}(-1)^{i} \frac{s^{r-i}}{(r-i+2) !} \mu_{(r-i+2)},
\end{aligned}
$$

where $\mu_{(r)}=E\left(X^{r}\right), \zeta(s)=E e^{-s X}$.

Proof. Since $F$ is NBRUL, then

$$
\int_{0}^{\infty} e^{-s x} \overline{W_{F}}(x+t) d x \leq \overline{W_{F}}(t) \int_{0}^{\infty} e^{-s x} \bar{F}(x) d x, \quad x, t \geq 0 .
$$

Making use of (2), yields

$$
\int_{0}^{\infty} t^{r} \int_{0}^{\infty} e^{-s x} \overline{W_{F}}(x+t) d x d t \leq \int_{0}^{\infty} t^{r} \overline{W_{F}}(t) \int_{0}^{\infty} e^{-s x} \bar{F}(x) d x d t .
$$

The left hand side of (3) can be written as

$$
\int_{0}^{\infty} t^{r} \overline{W_{F}}(t) \int_{0}^{\infty} e^{-s x} \bar{F}(x) d x d t=E \int_{0}^{\infty} t^{r} \overline{W_{F}}(t) \int_{0}^{\infty} e^{-s x} I(X>x) d x d t,
$$

where

$$
I(X>x)= \begin{cases}0 & \text { if } x \geq X \\ 1 & \text { if } x<X .\end{cases}
$$

After some calculations, the left hand side of (3) is given by

$$
\frac{\mu_{F}^{-1} \mu_{(r+2)}}{s(r+1)(r+2)}(1-\zeta(s)) \text {. }
$$

Also, the right hand side of (3) can be put in the following form

$$
\int_{0}^{\infty} t^{r} \overline{W_{F}}(t) \int_{0}^{\infty} e^{-s x} \bar{F}(x) d x d t=\int_{0}^{\infty} e^{-s v} \overline{W_{F}}(v) \int_{0}^{v} u^{r} e^{s u} d u d v .
$$

After some calculations (5) can be rewritten as

$$
\begin{aligned}
\int_{0}^{\infty} t^{r} \overline{W_{F}}(t) \int_{0}^{\infty} e^{-s x} \bar{F}(x) d x d t= & \frac{r !}{s^{r+1}} \mu_{F}^{-1} \sum_{i=0}^{r}(-1)^{i} \frac{s^{r-i}}{(r-i+2) !} \mu_{(r-i+2)} \\
& -\frac{(-1)^{r} r !}{s^{r+2}} \mu_{F}^{-1}\left[\mu_{F}-\frac{1}{s}(1-\zeta(s))\right] .
\end{aligned}
$$

From (4) and (6), Eq. (1) can be proved.

Remark. For $r=1$, Eq.(1) will be reduced to

$$
\frac{\mu_{3}}{6 s}[1-\zeta(s)] \leq \frac{1}{s^{3}}\left[\mu-\frac{1}{s}(1-\zeta(s))\right]+\frac{1}{s^{2}}\left[\frac{s}{6} \mu_{(3)}-\frac{1}{2} \mu_{(2)}\right],
$$

where $\mu_{(r)}=\int_{0}^{\infty} x^{r} d F(x)$.

\section{Testing against NBRUL alternatives}

Using Inequality (7) we can test the null hypothesis $H_{0}: F$ is exponential aganist $H_{1}: F$ is NBRUL and not exponential. $\delta_{1}(s)$ has been used as follows

$$
\delta^{(1)}(s)=\frac{1}{2 s^{2}} \mu_{(2)}-\frac{1}{6 s} \mu_{(3)} \zeta(s)-\frac{1}{s^{4}} \zeta(s)-\frac{1}{s^{3}} \mu+\frac{1}{s^{4}} .
$$


Note that under $H_{0}, \boldsymbol{\delta}^{(1)}(s)=0$, while under $H_{1}, \boldsymbol{\delta}^{(1)}(s)>0$.

Let $X_{1}, X_{2}, X_{3}, \ldots \ldots \ldots, X_{n}$ be a random sample from a distribution $F$. The empirical estimate $\delta_{n}^{(1)}(s)$ of $\delta^{(1)}(s)$ can be obtained as

$$
\delta_{n}^{(1)}(s)=\frac{1}{n^{2}} \sum_{i=1}^{n} \sum_{j=1}^{n}\left[\frac{1}{2 s^{2}} X_{i}^{2}-\frac{1}{6 s} X_{i}^{3} e^{-s X_{j}}-\frac{1}{s^{4}} e^{-s X_{i}}-\frac{1}{s^{3}} X_{i}+\frac{1}{s^{4}}\right] .
$$

To make the test invarient, let $\Delta_{n}^{(1)}(s)=\frac{\delta_{n}^{(1)}(s)}{\bar{X}^{4}}$, where $\bar{X}$ is the sample mean. Then

$$
\Delta_{n}^{(1)}(s)=\frac{1}{n^{2} \bar{X}^{4}} \sum_{i=1}^{n} \sum_{j=1}^{n}\left[\frac{1}{2 s^{2}} X_{i}^{2}-\frac{1}{6 s} X_{i}^{3} e^{-s X_{j}}-\frac{1}{s^{4}} e^{-s X_{i}}-\frac{1}{s^{3}} X_{i}+\frac{1}{s^{4}}\right]
$$

One can note that $\boldsymbol{\delta}^{(1)}(s)$ is an unbiased estimator of $\delta_{n}^{(1)}(s)$.

Now, set

$$
\phi_{s}\left(X_{i}, X_{j}\right)=\frac{1}{2 s^{2}} X_{i}^{2}-\frac{1}{6 s} X_{i}^{3} e^{-s X_{j}}-\frac{1}{s^{4}} e^{-s X_{i}}-\frac{1}{s^{3}} X_{i}+\frac{1}{s^{4}},
$$

and define the symmetric Kernel

$$
\psi_{s}\left(X_{i}, X_{j}\right)=\frac{1}{2 !} \sum_{R} \phi_{s}\left(X_{i}, X_{j}\right)
$$

where the summation is over all arrangements of $X_{i}, X_{j}$. Then $\Delta_{n}^{(1)}(s)$ in (9) is equivalent to the $\mathrm{U}_{n}$-statistic given by

$$
U_{n}=\frac{1}{\left(\begin{array}{c}
n \\
2
\end{array}\right)} \sum_{i<j} \psi_{s}\left(X_{i}, X_{j}\right)
$$

The asymptotic normality of $\Delta_{n}^{(1)}(s)$ can be summarized in the following theorem.

Theorem 4.1. (i) As $n \rightarrow \infty, \sqrt{n}\left(\Delta_{n}^{(1)}(s)-\Delta^{(1)}(s)\right)$ is asymptotically normal with mean 0 and variance $\sigma^{2}(s)$, where

$$
\begin{aligned}
\sigma^{2}(s)= & \operatorname{Var}\left\{\frac{1}{2 s^{2}} X^{2}-\frac{1}{6 s} X^{3} \zeta(s)-\frac{1}{s^{4}} e^{-s x}-\frac{1}{s^{3}} X+\frac{1}{2 s^{2}} \mu_{(2)}\right. \\
& \left.-\frac{1}{6 s} e^{-s x} \mu_{(3)}-\frac{1}{s^{4}} \zeta(s)-\frac{1}{s^{3}} \mu+\frac{2}{s^{4}}\right\}
\end{aligned}
$$

(ii) Under $H_{0}$, the variance $\sigma_{0}^{2}(s)$ is

$$
\sigma_{0}^{2}(s)=\frac{19+14 s+s^{2}}{(1+s)^{4}(1+2 s)}
$$

Proof. Using standard U-statistic theory, (Lee 1989),

$$
\sigma^{2}(s)=\operatorname{Var}\left\{E\left[\phi_{s}\left(X_{1}, X_{2}\right) \mid X_{1}\right]+E\left[\phi_{s}\left(X_{1}, X_{2}\right) \mid X_{2}\right]\right\}
$$

Recall the definition of $\phi_{s}\left(X_{i}, X_{j}\right)$ in (10), thus it is easy to show that

$$
E\left(\phi_{s}\left(X_{1}, X_{2}\right) \mid X_{1}\right)=\frac{1}{2 s^{2}} X^{2}-\frac{1}{6 s} X^{3} \int_{0}^{\infty} e^{-s x} d F(x)-\frac{1}{s^{4}} e^{-s x}-\frac{1}{s^{3}} X+\frac{1}{s^{4}},
$$

and

$$
\begin{aligned}
E\left(\phi_{s}\left(X_{1}, X_{2}\right) \mid X_{2}\right) & =\frac{1}{2 s^{2}} \int_{0}^{\infty} x^{2} d F(x)-\frac{1}{6 s} e^{-s x} \int_{0}^{\infty} x^{3} d F(x)-\frac{1}{s^{4}} \int_{0}^{\infty} e^{-s x} d F(x) \\
& -\frac{1}{s^{3}} \int_{0}^{\infty} x d F(x)+\frac{1}{s^{4}}
\end{aligned}
$$


therefore,

$$
\begin{aligned}
\sigma^{2}(s)= & \operatorname{Var}\left\{\frac{1}{2 s^{2}} X^{2}-\frac{1}{6 s} X^{3} \int_{0}^{\infty} e^{-s x} d F(x)-\frac{1}{s^{4}} e^{-s x}-\frac{1}{s^{3}} X+\frac{1}{2 s^{2}} \int_{0}^{\infty} x^{2} d F(x)\right. \\
& \left.-\frac{1}{6 s} e^{-s x} \int_{0}^{\infty} x^{3} d F(x)-\frac{1}{s^{4}} \int_{0}^{\infty} e^{-s x} d F(x)-\frac{1}{s^{3}} \int_{0}^{\infty} x d F(x)+\frac{2}{s^{4}}\right\}
\end{aligned}
$$

Under $\mathrm{H}_{0}$

$$
\sigma_{0}^{2}(s)=\frac{19+14 s+s^{2}}{(1+s)^{4}(1+2 s)}
$$

\section{The Pitman asymptotic efficiency}

To judge on the quality of this procedure, Pitman asymptotic efficiencies (PAEs) are computed and compared with some other tests for the following alternative distributions:

(i) The Weibull distribution: $\bar{F}_{1}(x)=e^{-x^{\theta}}, x \geq 0, \theta \geq 1$.

(ii) The linear failure rate distribution (LFR): $\bar{F}_{2}(x)=e^{-x-\frac{\theta}{2} x^{2}}, x \geq 0, \theta \geq 0$.

(iii) The Makeham distribution: $\bar{F}_{3}(x)=e^{-x-\theta\left(x+e^{-x}-1\right)}, x \geq 0, \theta \geq 0$.

Note that For $\theta=1, \bar{F}_{1}(x)$ reduces to exponential distribution while for $\theta=0, \bar{F}_{2}(x)$ and $\bar{F}_{3}(x)$ reduces to exponential distribution. The PAE is defined by:

$$
\operatorname{PAE}\left(\Delta_{n}^{(1)}(s)\right)=\frac{1}{\sigma_{\circ}(s)}\left|\frac{d}{d \theta} \delta_{\theta}^{(1)}(s)\right|_{\theta \rightarrow \theta_{\circ}} .
$$

At $s=5$,

$$
\delta_{\theta}^{(1)}(s)=\frac{1}{2 s^{2}} \mu_{\theta(2)}-\frac{1}{6 s} \mu_{\theta(3)} \zeta_{\theta}(s)-\frac{1}{s^{4}} \zeta_{\theta}(s)-\frac{1}{s^{3}} \mu_{\theta}+\frac{1}{s^{4}}
$$

where

$$
\begin{aligned}
\mu_{\theta} & =\int_{0}^{\infty} \bar{F}_{\theta}(u) d u, \mu_{\theta(2)}=2 \int_{0}^{\infty} u \bar{F}_{\theta}(u) d u, \mu_{\theta(3)}=3 \int_{0}^{\infty} u^{2} \bar{F}_{\theta}(u) d u \\
\zeta_{\theta}(s) & =E_{\theta}\left(e^{-s u}\right)=\int_{0}^{\infty} e^{-s u} d F_{\theta}(u)=-\int_{0}^{\infty} e^{-s u} d \bar{F}_{\theta}(u) .
\end{aligned}
$$

Hence,

$$
\frac{d}{d \theta} \delta_{\theta}^{(1)}(s)=\frac{1}{2 s^{2}} \mu_{\theta(2)}^{\prime}-\frac{1}{6 s}\left(\mu_{\theta(3)} \zeta_{\theta}^{\prime}(s)+\mu_{\theta(3)}^{\prime} \zeta_{\theta}(s)\right)-\frac{1}{s^{4}} \zeta_{\theta}^{\prime}(s)-\frac{1}{s^{3}} \mu_{\theta}^{\prime}
$$

where

$$
\begin{aligned}
1 & =\frac{d}{d \theta}, \mu_{\theta}^{\prime}=\int_{0}^{\infty} \bar{F}_{\theta}^{\prime}(u) d u, \mu_{\theta(2)}^{\prime}=2 \int_{0}^{\infty} u \bar{F}_{\theta}^{\prime}(u) d u, \\
\mu_{\theta(3)}^{\prime} & =3 \int_{0}^{\infty} u^{2} \bar{F}_{\theta}^{\prime}(u) d u, \zeta_{\theta}^{\prime}(s)=-\int_{0}^{\infty} e^{-s u} d \bar{F}_{\theta}^{\prime}(u) .
\end{aligned}
$$

Upon using the definition of the PAE in (14), we obtain

$$
\operatorname{PAE}\left(\delta^{(1)}\right)=\frac{1}{\sigma_{0}}\left|\frac{1}{2 s^{2}} \mu_{\theta(2)}^{\prime}-\frac{1}{6 s}\left(\mu_{\theta(3)} \zeta_{\theta}^{\prime}(s)+\mu_{\theta(3)}^{\prime} \zeta_{\theta}(s)\right)-\frac{1}{s^{4}} \zeta_{\theta}^{\prime}(s)-\frac{1}{s^{3}} \mu_{\theta}^{\prime}\right|_{\theta \rightarrow \theta_{0}} .
$$


Table 1: Comparison between the PAEs of our test and some other tests

\begin{tabular}{lccc}
\hline \multicolumn{1}{c}{ Test } & Weibull & LFR & Makeham \\
\hline (Kango 1993) & 0.132 & 0.433 & 0.144 \\
(Mugdadi and Ahmad 2005) & 0.170 & 0.408 & 0.039 \\
(Abdel Aziz 2007) & 0.223 & 0.535 & 0.184 \\
(Mahmoud and Abdul Alim 2002, 2003, 2008) & 0.050 & 0.217 & 0.144 \\
Our test $\Delta_{n}^{(1)}(5)$ & 1.046 & 0.932 & 0.233 \\
\hline
\end{tabular}

Table 2: Critical Values of the statistic $\Delta_{n}^{(1)}(5)$

\begin{tabular}{cccc}
\hline $\mathrm{n}$ & $90 \%$ & $95 \%$ & $99 \%$ \\
\hline 5 & 0.039527 & 0.051659 & 0.081158 \\
10 & 0.024073 & 0.029370 & 0.041747 \\
15 & 0.019395 & 0.023351 & 0.032516 \\
20 & 0.016674 & 0.019865 & 0.026928 \\
25 & 0.015041 & 0.017706 & 0.023554 \\
30 & 0.013927 & 0.016372 & 0.021441 \\
35 & 0.013179 & 0.015419 & 0.019853 \\
39 & 0.012648 & 0.014794 & 0.018750 \\
40 & 0.012483 & 0.014519 & 0.018562 \\
45 & 0.011991 & 0.013944 & 0.018084 \\
50 & 0.011490 & 0.013433 & 0.017040 \\
\hline
\end{tabular}

When $s=5$; this leads to

$$
\begin{gathered}
P A E\left[\Delta_{n}^{(1)}(5), \text { Weibull }\right]=1.04561, \operatorname{PAE}\left[\Delta_{n}^{(1)}(5), L F R\right]=0.931891 \text { and } \\
P A E\left[\Delta_{n}^{(1)}(5), \text { Makeham }\right]=0.232973 \text {, where } \sigma_{0}(5)=0.0894239 .
\end{gathered}
$$

From Table 1, it is obvious that $\Delta_{n}^{(1)}(5)$ is better than the other tests based on the PAEs.

\section{Monte Carlo null distribution critical points}

In this section the Monte Carlo null distribution critical points of $\Delta_{n}^{(1)}(5)$ are simulated based on 10000 generated samples of size $n=5(5) 35,39,40(5) 50$. From the standard exponential distribution by using Mathematica 8 program. Table 2 gives the upper percentile points of statistic $\Delta_{n}^{(1)}(5)$ for different confidence levels $90 \%, 95 \%$ and $99 \%$.

From Table 2, it is obvious that the critical values are decreasing as the samples size increasing and they are increasing as the confidence levels increasing.

\section{Power estimates of the test $\Delta_{n}^{(1)}(5)$}

In this section the power of our test $\Delta_{n}^{(1)}(5)$ will be estimated at $(1-\alpha) \%$ confidence level, $\alpha=0.05$ with suitable parameters values of $\theta$ at $n=10,20$ and 30 with respect to three alternatives Linear failure rate (LFR), Weibull and Gamma distributions based on 10000 samples.

Table 3 shows that the power estimates of our test $\Delta_{n}^{(1)}(5)$ are good power for all alternatives and increases when the value of the parameter $\theta$ and the sample sizes increasing. 


\begin{tabular}{ccccc}
\multicolumn{5}{c}{ Table 3: The Power Estimates of $\Delta_{n}^{(1)}(5)$} \\
\hline$n$ & $\theta$ & LFR & Weibull & Gamma \\
\hline 10 & 2 & 0.6674 & 0.9978 & 0.9922 \\
& 3 & 0.8501 & 1.0000 & 0.9991 \\
& 4 & 0.9324 & 1.0000 & 1.0000 \\
20 & 2 & 0.9360 & 1.0000 & 0.9888 \\
& 3 & 0.9816 & 1.0000 & 0.9988 \\
& 4 & 0.9911 & 1.0000 & 0.9998 \\
30 & 2 & 0.9828 & 1.0000 & 0.9861 \\
& 3 & 0.9944 & 1.0000 & 0.9992 \\
& 4 & 0.9983 & 1.0000 & 1.0000 \\
\hline
\end{tabular}

\section{Applications to real data}

In this section, we apply our test to some real data-sets at $95 \%$ confidence level.

1- Consider the data in (Al-Gashgari, Shawky, and Mahmoud 2016) which represent 39 liver cancers patients taken from Elminia cancer center Ministry of Health - Egypt, which entered in (1999). The ordered life times (in days)

$\begin{array}{cccccccccc}10 & 14 & 14 & 14 & 14 & 14 & 15 & 17 & 18 & 20 \\ 20 & 20 & 20 & 20 & 23 & 23 & 24 & 26 & 30 & 30 \\ 31 & 40 & 49 & 51 & 52 & 60 & 61 & 67 & 71 & 74 \\ 75 & 87 & 96 & 105 & 107 & 107 & 107 & 116 & 150 & \end{array}$

In this case, $\Delta_{n}^{(1)}(5)=0.0000132958$ which is less than the corresponding critical value in Table 2, then we reject $\mathrm{H}_{1}$ which states that the data set have NBRUL property.

2- Consider the real data-set given in (Grubbs 1971) and have been used in (Shapiro 1995). This data set gives the times between arrivals of 25 customers at a facility.

$\begin{array}{llllllllll}1.80 & 2.89 & 2.93 & 3.03 & 3.15 & 3.43 & 3.48 & 3.57 & 3.85 & 3.92 \\ 3.98 & 4.06 & 4.11 & 4.13 & 4.16 & 4.23 & 4.34 & 4.37 & 4.53 & 4.62 \\ 4.65 & 4.84 & 4.91 & 4.99 & 5.17 & & & & & \end{array}$

Since $\Delta_{n}^{(1)}(5)=0.00119843$ and this value less than the corresponding critical value in Table 2 . Then we conclude that this data set have the exponential property.

3- Consider the data in (Abouammoh et al. 1994). These data represent 40 patients suffering from blood cancer from one of the Ministry of Health Hospital in Saudi Arabia and the ordered life times (in days):

$\begin{array}{cccccccccc}115 & 181 & 255 & 418 & 441 & 461 & 516 & 739 & 743 & 789 \\ 807 & 865 & 924 & 983 & 1024 & 1062 & 1063 & 1169 & 1191 & 1222 \\ 1222 & 1251 & 1277 & 1290 & 1357 & 1369 & 1408 & 1455 & 1478 & 1549 \\ 1578 & 1578 & 1599 & 1603 & 1604 & 1696 & 1735 & 1799 & 1815 & 1852\end{array}$

Since $\Delta_{n}^{(1)}(5)=1.81685 \times 10^{-8}$ and this value less than the corresponding critical value in Table 2 . Then we conclude that this data set have the exponential property. 


\section{Conclusion}

The NBRUL class of life distributions is considered. The moments inequalities are derived. A new test statistics for exponentiality versus NBRUL class is proposed based on the moment inequalities. Quality criteria of the test is shown by the famous criterion which is Pitman asymptotic efficiency. The upper percentiles and the power of the proposed test are calculated and tabulated. Our test is applied to some real data to show the usefulness of the test.

\section{References}

Abdel Aziz A (2007). "On Testing Exponentiality Against RNBRUE Alternatives." Applied Mathematical Sciences, 35, 1725-1736.

Abouammoh A, Abdulghani S, Qamber I (1994). "On Partial Orderings and Testing of New Better Than Renewal Used Classes.” Reliability Engneering and System Safety, 43, 37-41.

Abouammoh A, Ahmed A (1988). "The New Better Than Used Class of Life Distribution.” Advances in Applied Probability, 20, 237-240.

Abouammoh A, Ahmed A (1992). “On Renewal Failure Rate Classes of Life Distributions.” Statistics and Probability Letters, 14, 211-217.

Abouammoh A, Newby J (1989). "On Partial Ordering the Tests of Generalized New Better Than Used Class of Life Distribution." Reliability Engneering and System Safety, 25, 207-217.

Abu-Youssef S (2009). "A Goodness of Fit Approach to Monotone Variance Residual Life Class of Life Distributions.” Applied Mathematical Sciences, 15, 715-724.

Ahmad I (1994). "Class of Statistics Useful in Testing Increasing Failure Rate Average and New Better than Used Life Distributions.” Journal of Statistical Plannng and Inference, 41, 141-149.

Ahmad I (2001). "Moments Inequalities of Aging Families of Distributions with Hypothesis Testing Applications." Journal of Statistical Plannng and Inference, 92, 121-132.

Ahmad I, Alwasel I, Mugdadi A (2001). "A Goodness of Fit Approach to Major Life Testing Problems." International Journal of Reliability Application, 2, 81-97.

Ahmad I, Mugdadi A (2004). "Further Moments Inequalities of Life Distributions with Hypothesis Testing Applications: the IFRA, NBUC, DMRL Classes." Journal of Statistical Plannng and Inference, 120, 1-12.

Al-Gashgari F, Shawky A, Mahmoud M (2016). "A Nonparametric Test for Testing Exponentiality against NBUCA Class of Life Distributions Based on Laplace Transform." Quality and Reliability Engineering International, 32, 29-36.

Atallah M, Mahmoud M, Al-Zahrani B (2014). "A New Test for Exponentiality versus NBUmgf Life Distributions Based on Laplace Transform." Quality and Reliability Engineering International, 30, 1353-1359.

Barlow R, Proschan F (1981). Statistical Theory of Reliability and Life Testing. To Begin with Silver Spring, M D.

Bryson M, Siddiqui M (1969). "Some Criteria for Ageing." Journal of the American Statistical Association, 64, 1472-1483.

Deshpande J, Kochar S, Singh H (1986). “Aspects of Positive Ageing.” Journal of Applied Probability, 23, 748-758. 
Grubbs F (1971). "Fiducial Bounds on Reliability for the Two Parameter Negative Exponential Distribution." Technometrics, 13, 873-876.

Ismail A, Abu-Youssef S (2012). "A Goodness of Fit Approach to the Class of Life Distribution with Unknown Age." Quality and Reliability Engineering International, 25, 761-766.

Kango A (1993). "Testing for New is Better than Used." Communications in Statistics-Theory and Methods, 12, 311-321.

Kumazawa Y (1983). “A Class of Tests Statistics for Testing whether New Is Better than Used." Communications in Statistics-Theory and Methods, 12, 311-321.

Lee A (1989). U-Statistics. Marcel Dekker, New York.

Mahmoud M, Abdul Alim N (2002). “On Testing Exponentiality Against NBARFR Life Distributions." Statistica, 4, 619-631.

Mahmoud M, Abdul Alim N (2003). "On Testing Exponentiality against NBURFR Class of Life Distributions." International Jornal of Reliability and Applications, 4, 57-69.

Mahmoud M, Abdul Alim N (2008). "A Goodness of Fit Approach to for Testing NBUFR (NWUFR) and NBAFR (NWAFR) Properties." International Jornal of Reliability and Applications, 9, 125140.

Mahmoud M, Diab L, Kayid M (2009). "Moments Inequalities for NBUL Distributions with Hypotheses Testing Applications." Contemporary Engineering Sciences, 7, 319-332.

Mahmoud M, EL-arishy S, Diab L (2003). "Moments Inequalities for Testing New Renewal Better than Used and Renewal New Better than Used Classes." International Journal of Reliability and Applications, 4, 97-123.

Mahmoud M, EL-Sagheer R, Etman W (2016). "Testing Exponentiality aganist New Better than Renewal Used in Laplace Transform Order." Journal of Statistics Applications \& Probability, 5(2), 279-285.

Mahmoud M, Moshref M, Mansour M (2015). "A New Class of Life Distributions with Hypotheses Testing Applications." Journal of Applied Probability and Statistics, 1, 35-47.

Mahmoud M, Rady D (2013). "Testing Exponentiality Against HNBUET Class Based on Goodness of Fit Approach." Al-Azhar Bulletin of Science, 24(1), 81-92.

Mugdadi A, Ahmad I (2005). "Moment Inequalities Derived from Comparing Life with Its Equilibrium Form.” Journal of Statistical Plannng and Inference, 134, 303-317.

Shapiro S (1995). Goodness of Fit Tests in The Exponential Distribution Theory Methods and Applications. Balakrishnan N, Basu AP. Editors, Gordon and Breach, Amsterdam. 


\section{Affiliation:}

Mohamed A. W. Mahmoud

Mathematics Department, Faculty of Science,

AL-Azhar University, Nasr City (11884),

Cairo, Egypt.

E-mail: mawmahmoudl1@azhar.edu.eg

R. M. EL-Sagheer

Mathematics Department, Faculty of Science, AL-Azhar University, Nasr City (11884),

Cairo, Egypt.

E-mail: rashadmath@azhar.edu.eg

W. B. H. Etman

Mathematics Department, Faculty of Science, AL-Azhar University, Nasr City (11884),

Cairo, Egypt.

E-mail: w.h.etman2012@gmail.com

\section{Austrian Journal of Statistics}

published by the Austrian Society of Statistics

Volume 47

February 2018 http://www.ajs.or.at/

http://www.osg.or.at/

Submitted: 2016-07-09

Accepted: 2016-08-19 